\title{
Bernstein's theorem on weighted Besov spaces
}

\section{HUY-QUI BUI}

Department of Mathematics, University of Canterbury,

Christchurch, New Zealand 


\title{
Bernstein's theorem on weighted Besov spaces
}

\author{
HUY-QUI BUI
}

\section{Introduction and preliminaries}

It is a well-known fact that the integrability of the Fourier transform of a function (or a distribution) is intimately related to its smoothness. The result of this type is usually called Bernstein's theorem. A most important result in this area is probably the Herz-Beurling theorem, which characterizes the Fourier transform of a distribution in the homogeneous Besov space $\dot{B}_{2, q}^{\alpha}$ (see [11]). The results of Herz [11] also improve the Hausdorff-Young theorem. Closely related to this latter theorem is the weighted estimate for the Fourier transform, which can be traced back to the theorem by Pitt and the uncertainty inequality. We refer to [13], [10], [1], [2], [8],[16], [17] for various such weighted estimates. In this note, which is one in a series of papers begun with [4] and devoted to a study of weighted function spaces, we propose to give a weighted version of Herz's results. The theorems we shall prove sharpen a number of weighted estimates mentioned above in the same manner as Herz's results did for the HausdorffYoung theorem. As an application of these theorems, we derive sufficient conditions for a function to be a multiplier on weighted Besov spaces with power weights. The results in this paper were announced in [7].

Next we shall define the function spaces needed in this paper. Let $\mathcal{S}\left(\mathbf{R}^{n}\right)=\mathcal{S}$ denote the Schwartz space of rapidly decreasing test functions and $\mathcal{S}^{\prime}\left(\mathbf{R}^{n}\right)=\mathcal{S}^{\prime}$ its dual, the space of tempered distributions. The Fourier transform of a distribution $f$ in $\mathcal{S}^{\prime}$ is denoted by $\hat{f}$ or $\mathcal{F} f$.

Choose a function $\theta$ in $\mathcal{S}$ with the following properties:

$$
\operatorname{supp} \hat{\theta} \subseteq\left\{\frac{1}{2} \leq|\xi| \leq 2\right\} ; \quad \sum_{j=-\infty}^{\infty} \hat{\theta}\left(2^{-j} \xi\right)=1,|\xi| \neq 0
$$

For each $j=0, \pm 1, \pm 2, \ldots$, let $\psi_{j}$ be the function in $\mathcal{S}$ given by $\hat{\psi}_{j}(\xi)=\hat{\theta}\left(2^{-j} \xi\right)$. Furthermore, let $\Phi \in \mathcal{S}$ be given by $\hat{\Phi}(\xi)+\sum_{j=1}^{\infty} \hat{\psi}_{j}(\xi)=1$ for all $\xi \in \mathbf{R}^{n}$.

Let $0<p<\infty, 0<q \leq \infty,-\infty<\alpha<\infty$, and $w$ be a weight function in the Muckenhoupt class $A_{\infty}$. Following J. Peetre [14], we define the weighted Besov spaces

$$
B_{p, q}^{\alpha, w}=\left\{f \in \mathcal{S}^{\prime}:\|f\|_{B_{p, q}^{\alpha, w}}=\|\Phi * f\|_{p, w}+\left(\sum_{j=1}^{\infty}\left(2^{j \alpha}\left\|\psi_{j} * f\right\|_{p, w}\right)^{q}\right)^{1 / q}<\infty\right\},
$$


and their homogeous versions,

$$
\dot{B}_{p, q}^{\alpha, w}=\left\{f \in \mathcal{S}^{\prime}:\|f\|_{\dot{B}_{p, q}^{\alpha, w}}=\left(\sum_{j=-\infty}^{\infty}\left(2^{j \alpha}\left\|\psi_{j} * f\right\|_{p, w}\right)^{q}\right)^{1 / q}<\infty\right\}
$$

where $\|g\|_{p, w}=\left(\int_{\mathbf{R}^{n}}|g(x)|^{p} w(x) d x\right)^{1 / p}$ is the (quasi-)norm on the weighted Lebesgue space $L_{w}^{p}$. We refer to [9] for properties of weight functions and to [4] for properties of the above weighted spaces. Note that the homogeneous spaces are defined modulo polynomials, i.e., they are embedded in $\mathcal{S}^{\prime} / \mathcal{P}$, the space of tempered distributions modulo polynomials.

We also let $H_{w}^{p}$ denote the weighted Hardy space of all tempered distributions $f$ for which

$$
\|f\|_{H_{w}^{p}}=\left\|\sup _{0<t<\infty}\left|\phi_{t} * f\right|\right\|_{p, w}<\infty
$$

where $\phi \in \mathcal{S}$ with $c_{\phi}=\int_{\mathbf{R}^{n}} \phi(x) d x \neq 0$, and $\phi_{t}(x)=t^{-n} \phi(x / t)$. Note that, if $f$ is a (measurable) function which has slow growth at infinity, i.e., there exists $N>0$ for which

$$
\int_{\mathbb{R}^{n}}|f(x)|(1+|x|)^{-N} d x<\infty
$$

then

$$
\|f\|_{p, w} \leq\left(1 /\left|c_{\phi}\right|\right)\|f\|_{H_{w}^{p}}
$$

by Lebesgue differentiation theorem.

Observe that, if $w \in A_{p}$ and $1<p \leq 2$, then we have the following continuous embeddings:

$$
B_{p, p}^{0, w} \subseteq L_{w}^{p}=H_{w}^{p} \subseteq B_{p, 2}^{0, w} .
$$

Similar results hold for homogeneous spaces if we interpret the embeddings modulo polynomials.

In order to describe the integrability of distributions in the weighted Besov spaces, we shall introduce a weighted version of a scale of function spaces defined by C.S. Herz (see [11], [12], [3]). Let $\mu$ be a non-negative, measurable function (on $\mathbf{R}^{n}$ ). Fix $0<\gamma<$ $\delta<\infty$, and let $0<p<\infty, 0<q \leq \infty$ and $-\infty<\alpha<\infty$. Define

$$
\begin{aligned}
K_{p, q}^{\alpha, \mu}=\{f: & \|f\|_{K_{p, q}^{\alpha, \mu}=}\left(\int_{\{|x| \leq \delta\}}|f(x)|^{p} \mu(x) d x\right)^{1 / p}+ \\
& \left.\left(\int_{1}^{\infty}\left(t^{\alpha p} \int_{\{\gamma t \leq|x| \leq \delta t\}}|f(x)|^{p} \mu(x) d x\right)^{q / p} \frac{d t}{t}\right)^{1 / q}<\infty\right\}
\end{aligned}
$$

(with the usual interpretation when $q=\infty$ ). Then $K_{p, q}^{\alpha, \mu}$ is a quasi-Banach space. Moreover, $K_{p, q}^{\alpha, \mu}$ does not depend on $\gamma$ or $\delta$, and for different values for $\gamma$ and $\delta$, the corresponding expressions on the right-hand side of the above give equivalent quasinorms. Below we shall list some further elementary properties of these spaces. 
(a) If $1<\rho \leq \lambda_{j+1} / \lambda_{j} \leq \sigma<\infty, j=1,2, \ldots$, then

$$
\begin{aligned}
\left(\int_{\left\{|x| \leq \lambda_{1}\right\}}|f(x)|^{p} \mu(x) d x\right)^{1 / p}+ & \\
& \left\{\sum_{j=1}^{\infty}\left(\int_{\left\{\lambda_{j} \leq|x| \leq \lambda_{j+1}\right\}}\left(|x|^{\alpha}|f(x)|\right)^{p} \mu(x) d x\right)^{q / p}\right\}^{1 / q}
\end{aligned}
$$

is an equivalent quasi-norm on $K_{p, q}^{\alpha, \mu}$, and $f \in K_{p, q}^{\alpha, \mu}$ if and only if $(1+|x|)^{\alpha} f \in K_{p, q}^{0, \mu}$.

(b) $K_{p, p}^{0, \mu}=L_{\mu}^{p}$ (equivalent norms).

(c) If $0<q \leq r \leq \infty$, then

$$
K_{p, q}^{\alpha, \mu} \subseteq K_{p, r}^{\alpha, \mu}
$$

(d) Assume that there exist $c>0, d>0$ and $t_{0}>0$ such that

$$
\int_{\{|x| \leq t\}} \mu(x) d x \leq c t^{d}
$$

for all $t \geq t_{0}$. Then

$$
K_{r, q}^{\alpha, \mu} \subseteq K_{p, q}^{\alpha-d(1 / p-1 / r), \mu}
$$

for all $0<p \leq r<\infty$

The following $C^{\infty}$-version of Lusin's theorem in measure theory seems standard, however, since we could not find it in the literature, we shall include a proof for easy reference. We follow [15] for terminologies in measure theory.

Proposition 1.1 Let $X$ be a smooth, n-dimensional manifold, and let $\mu$ be a nonnegative, complete, regular measure on a $\sigma$-algebra $\mathcal{M}$ in $X$, which contains all open subsets of $X$, such that $\mu(K)<\infty$ for all compact subsets $K$ in $X$. Assume that $s$ is a complex-valued simple function in $X$ such that

$$
\mu(\{x: s(x) \neq 0\})<\infty .
$$

Then for every $\epsilon>0$, there exists $g \in \mathcal{D}(X)$, the space of all infinitely differentiable functions on $X$ with compact supports, for which

$$
\begin{array}{r}
\mu(\{x: g(x) \neq s(x)\})<\epsilon, \\
\sup _{x \in X}|g(x)|=\sup _{x \in X}|s(x)| .
\end{array}
$$

Consequently, $\mathcal{D}(X)$ is dense in $L_{\mu}^{p}(X), 0<p<\infty$.

The results hold in particular when $X$ is an open subset of $\mathbf{R}^{n}, \mathcal{M}$ is the $\sigma$-algebra of Lebesgue measurable sets in $X$, and $d \mu(x)=w(x) d x$ for a non-negative locally integrable function $w$ on $X$.

PROOF. Let $s=\sum_{j=1}^{k} c_{j} \chi_{E_{j}}$, where each $c_{j} \neq 0$ is a complex number, each $E_{j} \in \mathcal{M}$ is non-empty with $\mu\left(E_{j}\right)<\infty$, and $E_{j} \cap E_{i}=\emptyset$ if $j \neq i$. Since $\mu$ is a regular measure, for each $j$ we can find a non-empty compact set $K_{j}$ and an open set $U_{j}$ such that

$$
\begin{array}{r}
K_{j} \subseteq E_{j} \subseteq U_{j}, \quad \mu\left(U_{j} \backslash K_{j}\right)<\epsilon / k, \\
U_{j} \cap U_{i}=\emptyset, \quad j \neq i .
\end{array}
$$


By a well-known result in differential calculus, there exists $g_{j} \in \mathcal{D}(X)$ with the following properties:

$$
0 \leq g_{j} \leq 1 ; \quad g_{j}=1 \text { on } K_{j} ; \quad \operatorname{supp} g_{j} \subseteq U_{j} .
$$

Put $g=\sum_{j=1}^{k} c_{j} g_{j}$. Then, clearly $g \in \mathcal{D}(X)$, and

$$
\begin{array}{r}
g(x)=c_{j}=s(x), \quad x \in K_{j}, \\
g(x)=0=s(x), \quad x \notin \cup_{j=1}^{k} U_{j} .
\end{array}
$$

It follows that

$$
\begin{array}{r}
\mu(\{x: g(x) \neq s(x)\}) \leq \sum_{j=1}^{k} \mu\left(U_{j} \backslash K_{j}\right)<\epsilon, \\
\sup _{x \in \mathbf{R}^{n}}|g(x)|=\max _{j}\left|c_{j}\right|=\sup _{x \in \mathbf{R}^{n}}|s(x)| .
\end{array}
$$

Since the set of simple functions is dense in $L_{\mu}^{p}(X), 0<p<\infty$, we deduce that $\mathcal{D}(X)$ is also dense in $L_{\mu}^{p}(X)$.

In the rest of this note we shall use $C$ to denote a positive constant which might change at each occurrence.

\section{Bernstein's theorem on inhomogeneous spaces}

Hereafter we use $\mathcal{F}$ to denote the Fourier transform.

Theorem 2.1 Let $1<p \leq r<\infty$ and $-\infty<\alpha<\infty$.

(i) Assume that $w \in A_{\infty}$, and $\mu$ is a non-negative measurable function such that there exists $M>0$ for which

$$
\int_{\mathbf{R}^{n}} \frac{\mu(\xi)^{1-r^{\prime}}}{(1+|\xi|)^{M}} d \xi<\infty
$$

If

$$
\|\mathcal{F} f\|_{r, \mu} \leq C\|f\|_{p, w}
$$

for all $f \in \mathcal{S}$, then

$$
\mathcal{F}: B_{p, q}^{\alpha, w} \rightarrow K_{r, q}^{\alpha, \mu}
$$

is bounded for all $0<q \leq \infty$.

(ii) Assume that $\mu \in A_{\infty}$, and $w$ is a non-negative measurable function such that there exists $N>0$ for which

$$
\int_{\mathbf{R}^{n}} \frac{w(x)^{1-p^{\prime}}}{(1+|x|)^{N}} d x<\infty
$$

If (2) holds for all $f \in \mathcal{S}$, then

$$
\mathcal{F}: K_{p, q}^{\alpha, w} \rightarrow B_{r, q}^{\alpha, \mu}
$$

is bounded for all $0<q \leq \infty$. 
Proof. We start with the proof of (i). Note that by (1), functions in $L_{\mu}^{r}$ have slow growth (at infinity), so that $L_{\mu}^{r} \subseteq \mathcal{S}^{\prime}$ (continuous embedding). Proposition 1.1 and (2) then imply that the Fourier transform $\mathcal{F}$ is uniquely extended to $L_{w}^{p}$, and (2) holds for every $f \in L_{w}^{p}$. (Note that, although $L_{w}^{p}$ may not be embedded in $\mathcal{S}^{\prime}, \mathcal{F} f$ has the usual meaning for $f \in \mathcal{S}^{\prime} \cap L_{w}^{p}$.) Let $f \in B_{p, q}^{\alpha, w}, W_{t}(x)=W(x, t)=(4 \pi t)^{-n / 2} e^{-|x|^{2} / 4 t}$ be the Gauss-Weierstrass kernel on $\mathbf{R}_{+}^{n+1}$, and $u(\cdot, t)=W_{t} * f$. By an argument similar to the proof of Lemma 4 in [3] and using the characterization of the weighted Besov spaces via temperatures in [5], it can be shown that, for each $t>0, \mathcal{F} f=\hat{f}=e^{t|\xi|^{2}} \hat{u}(\cdot, t)$ is a function having slow growth. Let $I_{j}=\left\{\xi: 2^{j-1} \leq|\xi| \leq 2^{j+1}\right\}, j= \pm 1, \pm 2, \ldots$, and let $I_{0}=\{\xi:|\xi| \leq 2\}$. Fix $j=1,2, \ldots$. Then, since

$$
\mathcal{F} f=\sum_{k=j-1}^{j+1} \mathcal{F}\left(\psi_{k} * f\right)
$$

in $I_{j}$, we deduce from (2) that

$$
\left(\int_{I_{j}}|\mathcal{F} f(\xi)|^{r} \mu(\xi) d \xi\right)^{1 / r} \leq C \sum_{j-1}^{j+1}\left\|\psi_{k} * f\right\|_{p, w}
$$

It follows that

$$
\begin{aligned}
\left\{\sum_{j=1}^{\infty}\left(\int_{I_{j}}\left(|\xi|^{\alpha}|\mathcal{F} f(\xi)|\right)^{r} \mu(\xi) d \xi\right)^{q / r}\right\}^{1 / q} & \approx\left\{\sum_{j=1}^{\infty}\left[2^{j \alpha}\left(\int_{I_{j}}|\mathcal{F} f(\xi)|^{r} \mu(\xi) d \xi\right)^{1 / r}\right]^{q}\right\}^{1 / q} \\
& \leq C\left(\sum_{j=0}^{\infty}\left(2^{j \alpha}\left\|\psi_{j} * f\right\|_{p, w}\right)^{q}\right)^{1 / q} \\
& \leq C\|f\|_{B_{p, q}^{\alpha, w} .}
\end{aligned}
$$

Since we have a similar estimate for $\left(\int_{I_{0}}|\mathcal{F} f(\xi)|^{r} \mu(\xi) d \xi\right)^{1 / r}$, we conclude that

$$
\|\mathcal{F} f\|_{K_{r, q}^{\alpha, \mu}}^{\alpha} \leq C\|f\|_{B_{,, q}^{\alpha, w}}
$$

We now turn to the proof of (ii). First note that by (3), functions in $K_{p, q}^{\alpha, w}$ have slow growth, so that $K_{p, q}^{\alpha, w} \subseteq \mathcal{S}^{\prime}$ and $\mathcal{F}$ is defined on $K_{p, q}^{\alpha, w}$. Choose the function $\theta$ in $\S 1$ such that $\hat{\theta}$ is radial; this implies that $\hat{\psi}_{j}(-\xi)=\hat{\psi}_{j}(\xi)$ for all $\xi$. Let $g \in K_{p, q}^{\alpha, w}$. Then

$$
\begin{aligned}
\left\|\psi_{j} * \hat{g}\right\|_{r, \mu} & =\left\|\mathcal{F}\left(\hat{\psi}_{j}(-\cdot) g\right)\right\|_{r, \mu} \\
& =\left\|\mathcal{F}\left(\hat{\psi}_{j} g\right)\right\|_{r, \mu} \\
& \leq C\left\|\hat{\psi}_{j} g\right\|_{p, w} \\
& \leq C\left(\int_{I_{j}}|g(x)|^{p} w(x) d x\right)^{1 / p}
\end{aligned}
$$

by (2). It follows that

$$
\left(\sum_{j=1}^{\infty}\left(2^{j \alpha}\left\|\psi_{j} * \hat{g}\right\|_{r, \mu}\right)^{q}\right)^{1 / q} \leq C\left\{\sum_{j=1}^{\infty}\left(\int_{I_{j}}\left(|x|^{\alpha}|g(x)|\right)^{p} w(x) d x\right)^{q / p}\right\}^{1 / q} .
$$


By a similar argument, we obtain

$$
\|\Phi * \hat{g}\|_{r, \mu} \leq C\left(\int_{I_{0}}|g(x)|^{p} w(x) d x\right)^{1 / p}
$$

The above two estimates imply the conclusion in (ii).

REMARK. There are many weighted estimates for the Fourier transform in the literature (see e.g. [2], [10], [13]), and our theorem can be used to sharpen these results. We just mention two such examples below.

In [13], B. Muckenhoupt proved that if $1<p \leq 2$, and there exists $A>0$ such that

$$
\sup _{t>0}\left(\int_{\{\mu(x)>A t\}} \mu(x) d x\right) \cdot\left(\int_{\left\{w(x)<t^{p-1}\right\}} w(x)^{1-p^{\prime}} d x\right)<\infty,
$$

then

$$
\|\mathcal{F} f\|_{p^{\prime}, \mu} \leq C\|f\|_{p, w} .
$$

If $w \in A_{p}$, then $L_{w}^{p} \subseteq B_{p, 2}^{0, w}$ (see $\S 1$ ). If $\mu$ also satisfies (1) with $r^{\prime}$ replaced by $p$, then $\mathcal{F}$ is bounded from $B_{p, 2}^{0, w}$ to $K_{p^{\prime}, 2}^{0, \mu}$. As the inclusions $L_{w}^{p} \subseteq B_{p, 2}^{0, w}$ and $K_{p^{\prime}, 2}^{0, \mu} \subseteq L_{\mu}^{p^{\prime}}$ are usually strict, our result sharpens Muckenhoupt's in this case.

Another example is the result by J. Benedetto, H. Heinig and R. Johnson ([2]), where they proved that, if $1<p \leq 2, w \in A_{p}$ is even on $\mathbf{R}^{\mathbf{1}}$, then

$$
\|\mathcal{F} f\|_{p, \mu} \leq C\|f\|_{p, w}
$$

where $\mu(\xi)=|\xi|^{p-2} w(1 / \xi), \xi \in \mathbf{R}^{1}$. (Note that $\mu \in A_{p}$ by a result of $\mathrm{R}$. Johnson and C. Neugebauer.) By our theorem $\mathcal{F}$ is bounded from $L_{w}^{p}=K_{p, p}^{0, w}$ to $B_{p, p}^{0, \mu}$. As $B_{p, p}^{0, \mu} \subseteq L_{\mu}^{p}$, our theorem improves the above result of Benedetto, Heinig and Johnson.

\section{Bernstein's theorem on homogeneous spaces}

To describe the integrability of distributions in $\dot{B}_{p, q}^{\alpha, w}$, we need to introduce the homogeneous versions of $K$-spaces

$$
\begin{aligned}
\dot{K}_{p, q}^{\alpha, \mu}= & \left\{f:\|f\|_{\dot{K}_{p, q}^{\alpha, \mu}}^{\alpha}=\right. \\
& \left.\left(\int_{0}^{\infty}\left(t^{\alpha p} \int_{\{\gamma t \leq|x| \leq \delta t\}}|f(x)|^{p} \mu(x) d x\right)^{q / p} \frac{d t}{t}\right)^{1 / q}<\infty\right\},
\end{aligned}
$$

where $\gamma, \delta, p, q$ and $\alpha$ are the same as in $\S 1$, and $\mu$ is a non-negative, measurable function. Then each $\dot{K}_{p, q}^{\alpha, \mu}$ is a quasi-Banach space. Moreover, when $\mu=1$, we shall drop $\mu$ from all notations. Note that properties similar to $(\mathrm{a})-(\mathrm{d})$ in $\S 1$ hold for the $\dot{K}$-spaces. For example, if $1<\rho \leq \lambda_{j+1} / \lambda_{j} \leq \sigma<\infty, j=0, \pm 1, \pm 2, \ldots$, then

$$
\left\{\sum_{j=-\infty}^{\infty}\left(\int_{\left\{\lambda_{j} \leq|x| \leq \lambda_{j+1}\right\}}\left(|x|^{\alpha}|f(x)|\right)^{p} \mu(x) d x\right)^{q / p}\right\}^{1 / q}
$$


is an equivalent quasi-norm on $\dot{K}_{p, q}^{\alpha, \mu}$.

When $p=\infty$, we also consider the unweighted Herz space $\dot{K}_{\infty, \infty}^{\alpha}$ equipped with the norm

$$
\begin{aligned}
\|f\|_{K_{\infty, \infty}^{\alpha}} & =\sup _{t>0} t^{\alpha}\left\|f \chi_{\{\gamma t \leq|x| \leq \delta t\}}\right\|_{\infty} \\
& \approx \sup _{j} \lambda_{j}^{\alpha}\left\|f \chi_{\left\{\lambda_{j} \leq|x| \leq \lambda_{j+1}\right\}}\right\|_{\infty} .
\end{aligned}
$$

For an open set $X$ in $\mathbf{R}^{n}$, as in $\S 1$, we use $\mathcal{D}(X)$ to denote the space of infinitely differentiable functions with compact supports in $X$, and $\mathcal{D}^{\prime}(X)$ its dual, the space of distributions in $X$.

Theorem 3.1 Let $1<p \leq r<\infty, 0<q \leq \infty$ and $-\infty<\alpha<\infty$.

(i) Assume that $w \in A_{\infty}, \mu$ is a non-negative measurable function for which $\mu^{1-r^{\prime}}$ is locally integrable on $\mathbf{R}^{n} \backslash\{0\}$. If

$$
\|\mathcal{F} f\|_{r, \mu} \leq C\|f\|_{H_{w}^{p}}
$$

for all $f \in \mathcal{S}$ such that $\hat{f} \in \mathcal{D}\left(\mathbf{R}^{n} \backslash\{0\}\right)$, then $\mathcal{F}$ is bounded from $\dot{B}_{p, q}^{\alpha, w}$ to $\dot{K}_{r, q}^{\alpha, \mu}$.

(ii) Assume that $\mu \in A_{\infty}$, and that $w$ is a non-negative measurable function such that there are real numbers $a, b$ for which

$$
\int_{\{|\xi|<1\}}|\xi|^{a} w(\xi)^{1-p^{\prime}} d \xi+\int_{\{|\xi| \geq 1\}}|\xi|^{b} w(\xi)^{1-p^{\prime}} d \xi<\infty
$$

If

$$
\|\mathcal{F} f\|_{r, \mu} \leq C\|f\|_{p, w}
$$

for all $f \in \mathcal{D}\left(\mathbf{R}^{n} \backslash\{0\}\right)$, then $\mathcal{F}$, appropriately defined, is bounded from $\dot{K}_{p, q}^{\alpha, w}$ to $\dot{B}_{r, q}^{\alpha, \mu}$.

Proof. We begin with the proof of (i). First, note that, since $\mu^{1-r^{\prime}}$ is locally integrable on $\mathbf{R}^{n} \backslash\{0\}$, functions in $L_{\mu}^{r}$ are also locally integrable there, so that $L_{\mu}^{r} \subseteq$ $\mathcal{D}^{\prime}\left(\mathbf{R}^{n} \backslash\{0\}\right)$ (continuous embedding).

Let $g \in H_{w}^{p}$. Then by [4, Lemma 4.4], there is a sequence $\left\{g_{j}\right\}$ in $\mathcal{S}$ such that each $\hat{g}_{j}=\mathcal{F} g_{j}$ is in $\mathcal{D}\left(\mathbf{R}^{n} \backslash\{0\}\right)$ and $g_{j} \rightarrow g$ in $H_{w}^{p}$ (and hence in $\mathcal{S}^{\prime}$ ). It follows from (4) that $\left\{\mathcal{F} g_{j}\right\}$ is a Cauchy sequence in $L_{\mu}^{r}$, so that there exists $h \in L_{\mu}^{r}$ for which

$$
\mathcal{F} g_{j} \rightarrow h
$$

in $L_{\mu}^{r}$ and hence in $\mathcal{D}^{\prime}\left(\mathbf{R}^{n} \backslash\{0\}\right)$. On the other hand

$$
\mathcal{F} g_{j} \rightarrow \mathcal{F} g
$$

in $\mathcal{S}^{\prime}$, and we deduce that

$$
\mathcal{F} g=h
$$

in $\mathcal{D}^{\prime}\left(\mathbf{R}^{n} \backslash\{0\}\right)$. Moreover, if $\mathcal{F} g=0$ in a neighbourhood of the origin, then $\mathcal{F} g=h$ in $\mathcal{S}^{\prime}$, and (4) holds for $g$, i.e.,

$$
\|\mathcal{F} g\|_{r, \mu} \leq C\|g\|_{H_{w}^{p}} .
$$


Let $f \in \dot{B}_{p, q}^{\alpha, w}$. Then each $\psi_{j} * f$ is in $H_{w}^{p}$ with $\mathcal{F}\left(\psi_{j} * f\right)=0$ in a neighbourhood of the origin, so that by the above observation we have

$$
\left\|\mathcal{F}\left(\psi_{j} * f\right)\right\|_{r, \mu} \leq C\left\|\psi_{j} * f\right\|_{H_{w}^{p}}
$$

for every $j$. Since

$$
\mathcal{F} f=\sum_{k=j-1}^{j+1} \mathcal{F}\left(\psi_{j} * f\right)
$$

in $I_{j}=\left\{2^{j-1} \leq|\xi| \leq 2^{j+1}\right\}$, a similar argument to the proof of (i) of Theorem 2.1 implies that

$$
\|\mathcal{F} f\|_{\dot{K}_{r, q}^{\alpha, \mu}} \leq C\|f\|_{\dot{B}_{p, q}^{\alpha, w}}
$$

Before proceeding on with the proof of (ii), let us extend the Fourier transform to $\dot{K}_{p, q}^{\alpha, w}$. (Note that $\dot{K}_{p, q}^{\alpha, w}$ may not be contained in $\mathcal{S}^{\prime}$, so the Fourier transform is not automatically defined for functions in $\dot{K}_{p, q}^{\alpha, w}$.) Let

$$
\mathcal{S}_{0}=\left\{\varphi \in \mathcal{S}: \int_{\mathbf{R}^{n}} x^{\kappa} \varphi(x) d x=0 \text { for every multi-index } \kappa\right\}
$$

Then

$$
\begin{gathered}
\hat{\mathcal{S}}_{0}=\mathcal{F}\left(\mathcal{S}_{0}\right)=\left\{\phi \in \mathcal{S}: D^{\kappa} \phi(0)=0 \text { for every multi-index } \kappa\right\}, \\
\mathcal{S}_{0}^{\prime}=\mathcal{S}^{\prime} / \mathcal{P},
\end{gathered}
$$

the space of tempered distributions modulo polynomials, and

$$
\hat{\mathcal{S}}_{0}^{\prime}=\mathcal{S}^{\prime} /\{\text { distributions supported at the origin }\} .
$$

Since $\mathcal{S}_{0}$ is dense in $\mathcal{S}_{0}^{\prime}$ and $\mathcal{F}$ is an isomorphism between $\mathcal{S}_{0}$ and $\hat{\mathcal{S}}_{0}$ (as Fréchet spaces), $\mathcal{F}$ is uniquely extended to an isomorphism between $\hat{\mathcal{S}}_{0}^{\prime}$ and $\mathcal{S}_{0}^{\prime}$.

Next we shall show that $\dot{K}_{p, q}^{\alpha, w}$ is continously embedded in $\hat{\mathcal{S}}_{0}^{\prime}$, so that $\mathcal{F}$ is defined on $\dot{K}_{p, q}^{\alpha, w}$. For this aim, let $f \in \dot{K}_{p, q}^{\alpha, w}$ and $\phi \in \hat{\mathcal{S}}_{0}$. Then, since $\phi \in \mathcal{S}$ has partial derivatives of all orders which vanish at the origin,

$$
|\phi(\xi)| \leq C|\xi|^{\alpha+1+a / p^{\prime}}
$$

for all $|\xi| \leq 2$, and

$$
|\phi(\xi)| \leq C|\xi|^{\alpha-1+b / p^{\prime}}
$$

for all $|\xi| \geq 1$. It follows that

$$
\begin{aligned}
& \left|\int_{\mathbf{R}^{n}} f(\xi) \phi(\xi) d \xi\right| \leq \\
& \quad \sum_{j=-\infty}^{0} \int_{I_{j}}|f(\xi)||\xi|^{\alpha} w(\xi)^{1 / p}|\xi|^{1+a / p^{\prime}} w(\xi)^{-1 / p} d \xi+\sum_{j=1}^{\infty} \int_{I_{j}}|f(\xi) \| \xi|^{\alpha} w(\xi)^{1 / p}|\xi|^{b / p^{\prime}-1} w(\xi)^{-1 / p} d \xi \\
& \quad \leq C\left\{\left(\int_{\{|\xi| \leq 2\}}|\xi|^{a} w(\xi)^{1-p^{\prime}} d \xi\right)^{1 / p^{\prime}}+\left(\int_{\{|\xi| \geq 1\}}|\xi|^{b} w(\xi)^{1-p^{\prime}} d \xi\right)^{1 / p^{\prime}}\right\}\|f\|_{\dot{K}_{p, \infty}^{\alpha, w}} \\
& \quad \leq C\|f\|_{\dot{K}_{p, q}^{\alpha, w}}
\end{aligned}
$$


by (5) and the embedding $\dot{K}_{p, q}^{\alpha, w} \subseteq \dot{K}_{p, \infty}^{\alpha, w}$. Hence $\dot{K}_{p, q}^{\alpha, w} \subseteq \hat{\mathcal{S}}_{0}^{\prime}$ as claimed.

The proof of (ii) is then completed in a way similar to the proof of of Theorem 2.1(ii).

REMARK. Besides the papers mentioned previously, there are others dealing with weighted estimates for functions with vanishing moments (see [1], [8], [16], [17]), and our theorem can be applied to the type of weight functions considered in these papers to improve a number of weighted estimates for the Fourier tranform contained therein, in a similar way to the Remark to Theorem 2.1.

\section{An application to multipliers}

We shall use the results obtained in previous sections to study multipliers on weighted Besov spaces. Though we shall deal only with homogeneous spaces, similar results hold for inhomogeneous ones, and precise formulations for the latter case are left to the interested reader.

First we recall the following Pitt's inequality, of which the result by Muckenhoupt is an extension.

PitT's Inequality. Assume that $1<p \leq r<\infty$, and

$$
0 \leq a<p-1, \quad b=\frac{r}{p}(a+1)-r+1 \geq 0 .
$$

Then

$$
\left(\int_{\mathbf{R}^{n}}|\hat{f}(\xi)|^{r}|\xi|^{-n b} d \xi\right)^{1 / r} \leq C\left(\int_{\mathbf{R}^{n}}|f(x)|^{p}|x|^{n a} d x\right)^{1 / p}
$$

By our Theorem 3.1, (8) implies that $\mathcal{F}$ is a bounded operator from $\dot{B}_{p, q}^{\alpha,|x|^{n a}}$ to $\dot{K}_{r, q}^{\alpha,|x|^{-n b}}$ for all $\alpha$ and all $q$. Let $\beta$ and $c$ be real numbers, and $m$ and $g$ be measurable functions. Assume that $p<r$. Then, with $s=p r /(r-p)$, Hölder's inequality implies that

$$
\left(\int_{I_{j}}|m(x) g(x)|^{p}|x|^{n c} d x\right)^{1 / p} \leq C \lambda_{j}^{n e}\left(\int_{I_{j}}|g(x)|^{r}|x|^{-n b} d x\right)^{1 / r}\left(\int_{I_{j}}|m(x)|^{s} d x\right)^{1 / s}
$$

where $e=c / p+b / r, I_{j}=\left\{\lambda_{j} \leq|x| \leq \lambda_{j+1}\right\}$, and $1<\rho \leq \lambda_{j+1} / \lambda_{j} \leq \sigma<\infty$ for $j=0, \pm 1, \pm 2, \ldots$. It follows that

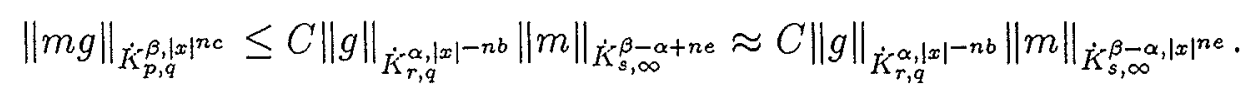

If $p=r$, we similarly obtain the inequality

$$
\|m g\|_{\dot{K}_{p, q}^{\beta,|x|^{n c}}} \leq C\|g\|_{\dot{K}_{r, q}^{\alpha,|x|}-n b}\|m\|_{\dot{K}_{\infty, \infty}^{\beta-\alpha+n e}}
$$

Next assume that

$$
0 \leq d<1, \quad c=\frac{p}{r}(d-1)+p-1 \geq 0 .
$$

Then Pitt's inequality and our Theorem 3.1 imply that $\mathcal{F}^{-1}$ is bounded from $\dot{K}_{p, q}^{\beta,|x|^{n c}}$ to $\dot{B}_{r, q}^{\beta,|x|^{-n d}}$. Since $c / p+b / r=a / p+d / r$ by (7) and (9), we have proved the following theorem. 
Theorem 4.1 Assume that $1<p \leq r<\infty$, and a,d are non-negative real numbers for which (7) and (9) hold. Put $s=p r /(r-p)$ and $e=a / p+d / r$. Then

$$
f \quad \mapsto \quad \mathcal{F}^{-1}(m \hat{f})
$$

is a bounded operator from $\dot{B}_{p, q}^{\alpha,|x|^{n a}}$ into $\dot{B}_{r, q}^{\beta,|x|^{-n d}}$, for any $m \in \dot{K}_{s, \infty}^{\beta-\alpha+n e}=\dot{K}_{s, \infty}^{\beta-\alpha,|x|^{n e}}$, and for all $\alpha, \beta$ and $q$. Moreover, the operator norm is dominated by a constant multiple of the $\dot{K}$-norm of $m$.

REMARK. The results in Theorem 4.1 complement those in [6].

\section{References}

[1] J. Benedetto and H. Heinig, Fourier transform inequalities with measure weights, Adv. in Math. 96(1992), 194-225.

[2] J. Benedetto, H. Heinig and R. Johnson, Fourier inequalities with $A_{p}$-weights, in "General inequalities 5" (W. Walter, Ed.), pp.217-232, Birkhäuser, Basel, 1987.

[3] H.-Q. Bui, Bernstein's theorem and translation invariant operators, Hiroshima Math. J. 11(1981), 81-96.

[4] H.-Q. Bui, Weighted Besov and Triebel spaces: Interpolation by the real method, Hiroshima Math. J. 12(1982), 581-605.

[5] H.-Q. Bui, Characterizations of weighted Besov and Triebel-Lizorkin spaces via temperatures, J. Functional Anal. 55(1984), 39-62.

[6] H.-Q. Bui, Weighted Young's inequality and convolution theorems on weighted Besov spaces, Math. Nachr. 170(1994), 25-37.

[7] H.-Q. Bui, Recent results in weighted function spaces, in "Seminar in Harmonic Analysis 9 (1993) (edited by S. Igari)", pp. 44-54.

[8] C. Carton-Lebrun, Fourier inequalities with nonradial weights, Trans. Amer. Math. Soc. 333(1992), 751-767.

[9] J. García-Cuerva and J.L. Rubio de Francia, Weighted norm inequalities and related topics, North Holland, Amsterdam, New York, 1985.

[10] H. Heinig, Weighted norm inequalities for classes of operators, Indiana Math. J. $33(1984), 573-582$.

[11] C.S. Herz, Lipschitz spaces and Bernstein's theorem on absolutely convergent Fourier transforms, J. Math. Mech. 18(1968), 283-324.

[12] R. Johnson, Lipschitz spaces, Littlewood-Paley spaces, and convoluteurs, Proc. London Math. Soc. 29(1974), 127-141. 
[13] T. Mizuhara, On some function spaces and Lipschitz spaces, J. London Math. Soc. 25(1982), 75-87.

[14] B. Muckenhoupt, Weighted norm inequalities for the Fourier transform, Trans. Amer. Math. Soc. 276(1983), 729-742.

[15] J. Peetre, New thoughts on Besov spaces, Duke University Mathematics Series, Durham, 1976.

[16] W. Rudin, Real and complex analysis, McGraw-Hill, New York, 1966.

[17] C. Sadosky and R.L. Wheeden, Some weighted norm inequalities for the Fourier transform of functions with vanishing moments, Trans. Amer. Math. Soc. 300(1987), 521-533.

[18] J-O. Strömberg and R.L. Wheeden, Weighted norm estimates for the Fourier transform with a pair of weights, Trans. Amer. Math. Soc. 318(1990), 355-372.

Department of Mathematics \& Statistics,

University of Canterbury,

Christchurch 1, New Zealand.

E-mail: hqb@math.canterbury.ac.nz 